\title{
Controlling Energy-Level Alignments at Carbon Nanotube/Au Contacts
}

\author{
Xiaodong Cui, ${ }^{\dagger}$ Marcus Freitag, ${ }^{\ddagger}$ Richard Martel,, Louis Brus, ${ }^{\dagger}$ and \\ Phaedon Avouris $\mathbf{s}^{*,+, \$}$
}

Chemistry Department, Columbia University, New York, New York 10027,

Carbon Nanotechnologies Inc., 16200 Park Row, Houston, Texas 77084, and

IBM Research Division, T.J. Watson Research Center,

Yorktown Heights, New York 10598

Received April 1, 2003

\begin{abstract}
The properties of the contacts between single-walled carbon nanotubes (SWNTs) and Au electrodes are studied using scanning Kelvin probe and electrostatic force microscopies. Contact potential differences and local dipoles at the SWNT/Au interface are determined under various conditions involving gas adsorption and surface passivation. In particular, the effects of the coadsorption of alkanethiol, $\mathrm{S}$, and $\mathrm{O}_{2}$ are explored in detail. We find that the coadsorbates alter the energy-level line-up at the contacts and induce significant shifts of the SWNT bands relative to the metal Fermi level. This behavior is explained by considering the response of the local Au work function to the presence of the nanotube and of the coadsorbates as well as the effects of the adsorbate dipoles near the contacts. Finally, we use coadsorption to control the Schottky barrier height at the nanotube-Au contacts.
\end{abstract}

Single-walled carbon nanotubes (SWNTs) are prototypical 1D quantum wires whose electronic properties depend strongly on their diameter and chirality. Thus, SWNTs can be metallic or semiconducting with a band gap that is inversely proportional to their diameter. Scattering and therefore power dissipation are drastically reduced, and their strong covalent bonding allows them to withstand extremely high current densities. ${ }^{1-3}$ A number of SWNT-based electronic devices have been demonstrated-most importantly, high-performance SWNT field-effect transistors (SWNTFETs). ${ }^{4-6}$

In the first studies of SWNT-FETs, ${ }^{4,5}$ it was observed that they operate as p-type (i.e., hole transport) devices in air, even though they were not intentionally doped. It was later found, however, that in ultrahigh vacuum (UHV) they behaved as n-type (i.e., electron transport) devices. ${ }^{7,8}$ Initially, the $\mathrm{p}$ character of SWNT-FETs in air was ascribed to hole doping by charge transfer from the SWNT to atmospheric $\mathrm{O}_{2}{ }^{9,10}$ However, if the $\mathrm{p}$ character of SWNT-FETs in air is due to oxygen doping, then in vacuum the SWNTs should be undoped, and therefore the SWNT-FETs should be ambipolar, not $\mathrm{n}$ type as is observed. Recently, however, strong evidence has been presented that the metal-nanotube Schottky barriers determine the electrical character of the SWNT-FETs, ${ }^{711-13}$ and that the transistor switching mech-

\footnotetext{
* Corresponding author. E-mail: avouris@us.ibm.com.

Columbia University.

$\div$ Carbon Nanotechnologies Inc.

$\S$ IBM Research Division.
}

anism in SWNT devices is fundamentally different from that of conventional Si FETs. Specifically, it was concluded that SWNT-FETs are 1D Schottky barrier devices in which the modulation of the carrier injection barrier, instead of the channel conductance, is the basis of the transistor action. ${ }^{11-15}$ In this model, oxygen adsorption on the metal electrode/ SWNT interface affects the alignment of the nanotube bands with respect to the metal electrode Fermi level, thus altering the carrier injection barriers. As a result, holes are easily injected in air, and electron transport is favored in vacuum. It is clear then that optimizing the performance of SWNTFETs requires a better understanding of the interactions occurring at the SWNT/metal contacts. Unlike the interfaces between metals and bulk inorganic semiconductors, such junctions are poorly understood.

Here we report studies of SWNT/Au interfaces with scanning Kelvin probe microscopy ${ }^{16}$ (SKPM) and electrostatic force microscopy (EFM). ${ }^{17}$ The results indicate that the interfacial dipole layer at SWNT/Au junctions reversibly changes direction as the environment changes from ambient air to vacuum or an oxygen-free environment. Consequently, the Au Fermi level lies above the SWNT mid-gap in oxygenfree environments and below the mid-gap in air. In contrast, there is no significant dependence of the energy-level alignment at SWNT/graphite (HOPG) interfaces on oxygen. Furthermore, on $\mathrm{Au}$, the energy-level alignment is strongly sensitive to the coadsorption of other molecular absorbates such as $\mathrm{H}_{2} \mathrm{~S}$ or alkanethiol self-assembled monolayers (SAMs). In this way, we obtain a passivation of the contacts 

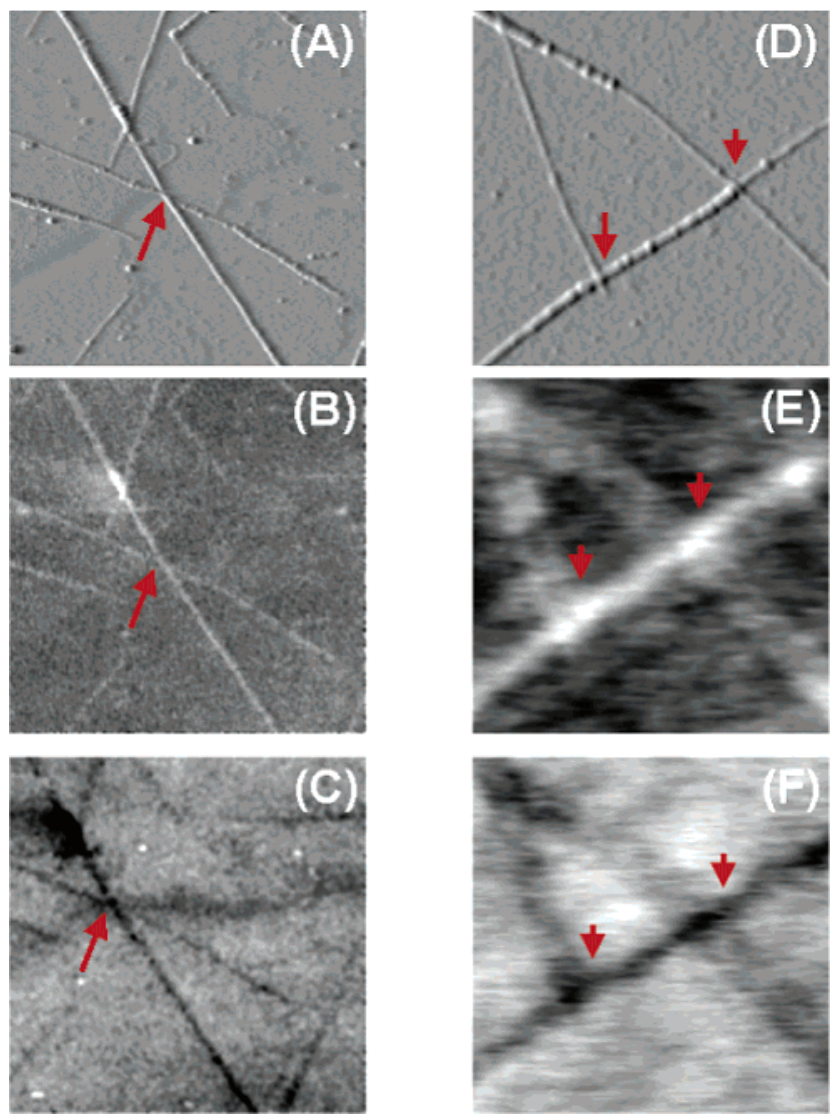

Figure 1. In situ atomic force microscopy (AFM), scanning Kelvin probe microscopy (SKPM), and electrostatic force microscopy (EFM) images of SWNTs on Au(111) substrates in controlled environments. (A, D) AFM topographic images of nanotubes deposited on flame-annealed Au surfaces. (B, C) SKPM images obtained in air and after a long period in ultrahigh vacuum (UHV), respectively. Bright contrast indicates a lower vacuum level. (E, F) In situ EFM images taken in air and in an ambient $\mathrm{N}_{2}$ environment, respectively. Bright contrast in the EFM images indicates that the interfacial dipole is pointing toward the substrate.

against further oxygen adsorption and a permanent modification of the carrier injection barriers, which provides a way of tuning the transport properties of SWNT-FETs. Examples in which we enhance the performance of p-type SWNT-FETs or convert a unipolar p-type SWNT-FET to an ambipolar one are presented. These results provide further proof of the Schottky barrier model for the SWNT-FETs.

Experimental Section. The semiconducting SWNTs used in our experiments were provided by Rice University. They have an average diameter of $1.4 \mathrm{~nm}^{18}$ and a band gap of about $700 \mathrm{meV} \cdot{ }^{19,20}$ Samples for SKPM and EFM experiments were obtained by dispersing SWNT solutions in dichloroethane onto three different substrates: polycrystalline $\mathrm{Au}$ on $\mathrm{SiO}_{2}$, flame-annealed $\mathrm{Au}(111)$ on mica (Molecular Imaging), and freshly cleaved highly oriented pyrolytic graphite (HOPG). The SKPM experiments were carried out on a JEOL A4500 microscope using $\mathrm{Pt}, \mathrm{W}_{2} \mathrm{C}$-coated, or $\mathrm{n}^{+}$Si conducting AFM tips in a UHV chamber $\left(\sim 10^{-7} \mathrm{~Pa}\right)$ or in air. In the Kelvin probe images (see Figure 1B and C), a bright region indicates a locally lower vacuum level or surface potential. EFM measurements were performed using a Nanoscope III DI microscope with Pt-coated tips (Nanosensor) either in a $\mathrm{N}_{2}$-filled glovebox $\left(\mathrm{O}_{2} \approx 1 \mathrm{ppm}\right)$ or in air. In EFM images (Figure $1 \mathrm{E}$ and $\mathrm{F}$ ), the brighter features indicate positive charges or dipoles pointing toward the bulk of the sample ( $\downarrow$ ). In SKPM and EFM measurements, individual SWNT tubes or bundles of tubes were first identified by AFM topographic images. The charge distribution and charge density on SWNTs were deduced by fitting the curves of the first harmonic component of EFM signals, the force gradient, as a function of the tip-sample distance..$^{21}$

SKPM measures the difference between the local work function of the sample and that of the reference SKPM tip, which is made of $n^{+} \mathrm{Si}$ with nanometer resolution. The EFM measurement, however, provides a measure of the charge transfer that takes place at the nanotube-metal interface. Thus, the SKPM and EFM measurements provide unique information about the electronic structure and interactions at the interface.

SWNT-FETs were fabricated as described elsewhere, ${ }^{11}$ a $\mathrm{p}^{++}$-doped silicon wafer with a 120 -nm-thick $\mathrm{SiO}_{2}$ layer was used as a back gate, and the Au source and drain electrodes separated by $500 \mathrm{~nm}$ were built on top of it. Passivating monolayers of $\mathrm{S}$ atoms on the $\mathrm{Au}$ surface were formed by exposing $\mathrm{Au}$ substrate/electrodes to gaseous $\mathrm{H}_{2} \mathrm{~S}$ for 5-10 $\min ^{22}$ before dispersing the SWNTs. In the case of alkanethiol-modified samples, we first disperse SWNTs on the Au substrates/FET chip and incubate them in a $4 \mathrm{mM} \mathrm{C}_{16} \mathrm{H}_{34^{-}}$ $\mathrm{SH}$ alkanethiol solution in ethanol for over $8 \mathrm{~h}$. We then copiously rinse the samples with ethanol and finally anneal them at $80{ }^{\circ} \mathrm{C}$ for $1 \mathrm{~h}$. All electrical measurements are carried out in air with a semiconductor parameter analyzer (HP4145).

Results. Typical AFM, SKPM, and EFM images of SWNTs on $\mathrm{Au}(111)$ in air and in vacuum are shown in Figure 1. Contact potential differences (CPDs) and information on interface dipoles are summarized in Table 1. The CPDs between HOPG substrates and individual SWNTs are found to be about $-0.1 \mathrm{eV}$ in both ultrahigh vacuum (UHV, $10^{-8}-10^{-9}$ Torr) and in ambient air. Given the HOPG work function of $4.6 \mathrm{eV},{ }^{23}$ a SWNT work function of $4.7 \mathrm{~V}$ is deduced by adding the HOPG work function and the CPD. This value is in agreement with the reported value of 4.8 $\mathrm{eV}$ obtained by UPS.$^{23}$ It is important to note that the CPDs between adsorbed SWNTs on HOPG and HOPG itself are essentially the same in air and in UHV. That is, the energylevel alignment at the SWNT/HOPG interface remains the same in both environments. It is known that oxygen weakly physisorbs with negligible charge transfer on structurally perfect HOPG. ${ }^{24,25}$ Therefore, we conclude that oxygen does not dope the semiconductor SWNTs either.

As shown in Table 1, the CPDs between the Au substrate and the adsorbed SWNTs are about $-0.06 \mathrm{~V}$ for $\mathrm{Au}(111)$ and $-0.08 \mathrm{~V}$ for polycrystalline $\mathrm{Au}$ in air, whereas they are $0.05 \mathrm{~V}$ for $\mathrm{Au}(111)$ and $0.19 \mathrm{~V}$ for polycrystalline $\mathrm{Au}$ in UHV. These values are significantly different from the values deduced by comparing the differences in the tabulated work functions of the individual materials. For example, $\Phi_{\mathrm{Au}}-$ $\Phi_{\text {HOPG }}>0.5 \mathrm{eV} .{ }^{26,27}$ It is well known, however, that the work function of Au surfaces is very sensitive to contami- 
Table 1. Contact Potential Differences $\left(\Delta \Phi_{\mathrm{s}}=\Phi_{\text {substrate }}-\Phi_{\mathrm{SWNT} / \mathrm{substrate}}\right)$ and Interfacial Dipoles ${ }^{a}$

\begin{tabular}{lcccc}
\hline & \multicolumn{2}{c}{ air } & \multicolumn{2}{c}{ UHV } \\
\cline { 2 - 5 } & CPD (V) & interfacial & CPD (V) & interfacial \\
dipole & & dipole & $\uparrow$ \\
SWNT/Au(poly) & $0.08 \pm 0.03$ & $\downarrow$ & $-0.19 \pm 0.05$ & $\uparrow$ \\
SWNT/Au(111) & $0.06 \pm 0.02$ & $\downarrow$ & $-0.05 \pm 0.02$ & $\uparrow$ \\
SWNT/HOPG & $-0.09 \pm 0.02$ & $\uparrow$ & $-0.11 \pm 0.02$ & $\uparrow$ (weak) \\
thiol/SWNT/Au(111) & $-0.05 \pm 0.02$ & $\uparrow$ (weak) & $-0.03 \pm 0.02$ & negligible
\end{tabular}

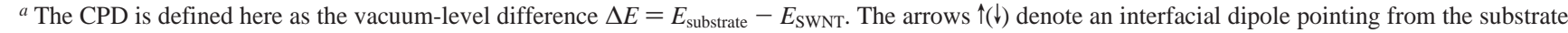
(SWNT) to the SWNT (the substrate). These dipoles are barely detectable at the interfaces of SWNTs on sulfur-passivated Au and at interfaces of alkanethiolmodified SWNT/Au.

nants. Exposure to air invariably reduces the work function, primarily because of hydrocarbon adsorption, to values as low as $4.5 \mathrm{~V} .{ }^{27}$ It is important to note that, in our nanotube studies, the SWNT-baring Au surfaces have also been exposed to the solvent during SWNT deposition.

The CPD measurements in Table 1 indicate that the energy-level alignment at SWNT/Au interfaces is strongly sensitive to the presence of oxygen. As shown in Figure 1, the vacuum level of the SWNT is lower than that of $\mathrm{Au}$ in air but is higher in UHV. EFM measurements indicate that the interface dipole at the SWNT/Au interface points from SWNT(+) to $\mathrm{Au}(-)$ in air but reverses its direction in UHV or in a clean $\mathrm{N}_{2}$ atmosphere. At room temperature, this transformation is slow and requires from 1 or $2 \mathrm{~h}$ to a few days depending on the crystallinity and morphology of the Au substrate. Most importantly, the processes are reversible. From the EFM measurements, we deduce that the SWNTs on $\mathrm{Au}$ are negatively charged with a line density of about $0.1-0.05 \mathrm{e} / \mathrm{nm}$ in oxygen-free environments but positively charged with approximately 1e/nm in air. Thus, the Au Fermi level lies above the SWNT mid-gap in vacuum or in inert gases and below the mid-gap in air. The energy-level alignments at the SWNT/Au(111) interfaces deduced from SKPM and EFM experiments are shown diagrammatically in Figure 2. These findings are in full agreement with the electrical transport results, which show p-type SWNT-FET characteristics in air $^{4,5}$ and n-type characteristics in UHV or inert gases. ${ }^{7,8}$

To understand the above results, we need to consider in detail the interactions involved. SWNTs are thought to bind to Au surfaces by van der Waals forces, and no significant charge transfer between Au and SWNTs is expected on the basis of the energies of the bands of free nanotubes. However, the nanotube-metal interaction can lead to significant changes in the electronic structures of both the SWNT and the metal surface. First, we note that the van der Waals binding of nanotubes to substrates is unusually strong. ${ }^{28}$ The close proximity of the SWNT to the Au surface leads to a Born repulsion between the closed-shell semiconducting nanotube and the sp "spill-out" electrons of $\mathrm{Au}$, which contribute most to the Au surface dipole and control its work function. By pushing back the spill-out charge, the SWNT locally generates an interface dipole opposed to the surface dipole of clean Au and locally reduces the Au work function. ${ }^{27,29}$ (Similar effects would be induced by any

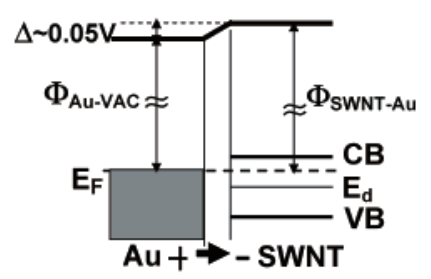

(A)
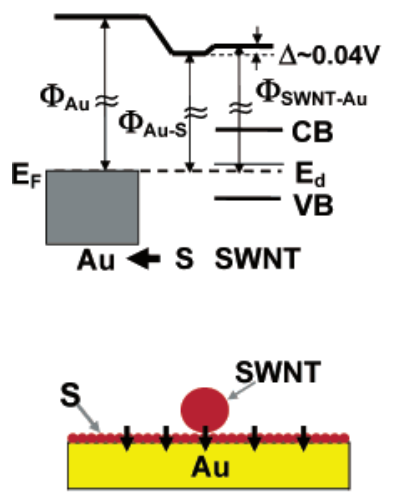

(C)

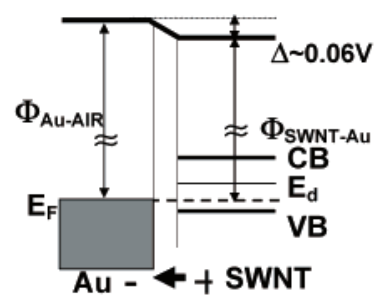

(B)
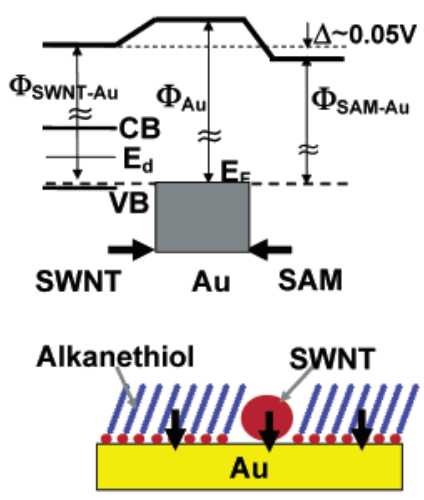

(D)
Figure 2. Diagrams showing the energy-level alignments at (A) single-walled carbon nanotube (SWNT)/Au interfaces in air, (B) SWNT/Au in UHV, (C) SWNT/S-Au, and (D) alkanethiolmodified SWNT/Au surface.

reaction products of the dichloroethane solvent with the surface.) Furthermore, the SWNT valence and conduction bands (van Hove singularities) are broadened $(\Gamma>0.1 \mathrm{eV})$ by the interaction with the Au surface. ${ }^{30}$ Thus, charge transfer from Au to the broadened SWNT levels leads to a lowering of their energy and an increased binding energy because of charge-image charge interactions. The interactions described above can account for the Au-to-SWNT charge transfer observed in the absence of oxygen. Similar effects have been observed in the interactions of $\mathrm{C}_{60}{ }^{29}$ and conjugated organic molecules ${ }^{31}$ with $\mathrm{Au}$.

On going from an oxygen-free environment to an ambient oxygen environment, we find that the work function of the $\mathrm{Au}$ substrates increases by $\gtrsim 0.1 \mathrm{eV}$. This finding can be understood by noting that, upon adsorption of $\mathrm{O}_{2}$, a partial charge transfer from the metal to $\mathrm{O}_{2}$ is expected. This charge transfer creates an interface dipole in the same direction as 

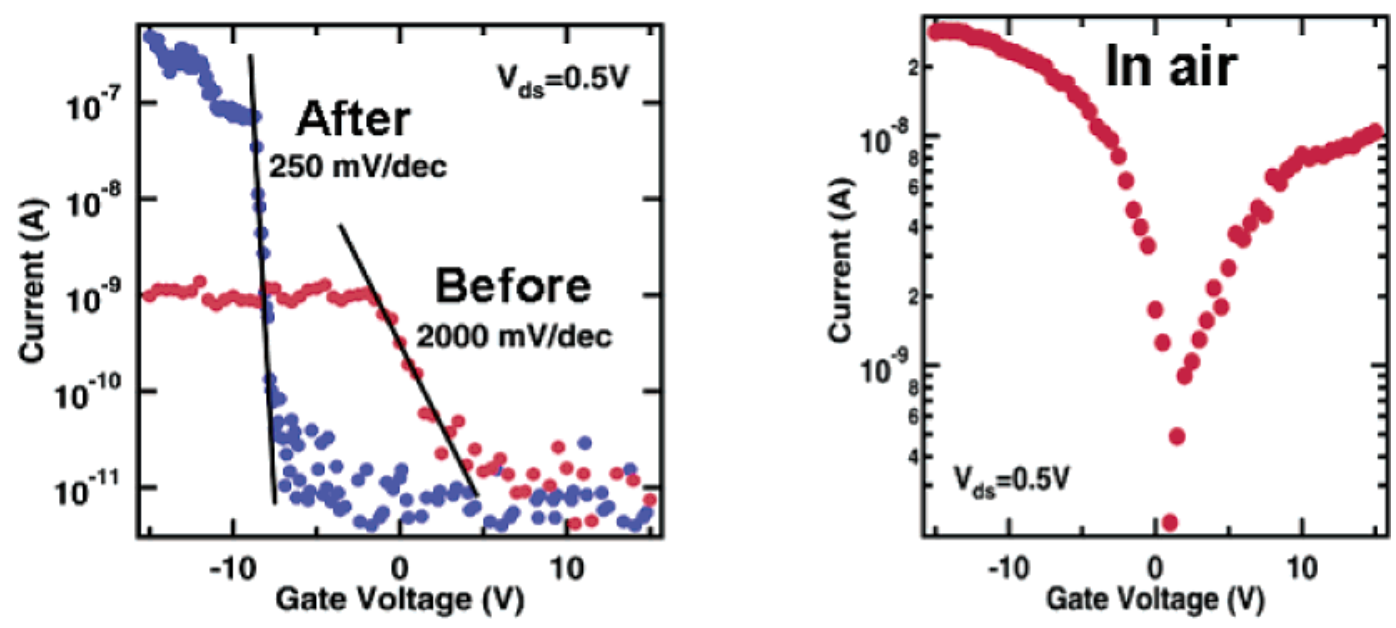

Figure 3. (Left) Drain current vs gate-voltage curves ( $I_{\mathrm{ds}}$ vs $\left.V_{\mathrm{g}}\right)$ of a SWNT-FET with a clean gold source and drain contacts in air (red circles) and after the deposition of a $\mathrm{C}_{16} \mathrm{H}_{34} \mathrm{SH}$ alkanethiol self-assembled layer (blue circles). (Right) $I_{\mathrm{ds}}$ vs $V_{\mathrm{g}}$ curve of an ambipolar SWNT-FET fabricated with sulfur/Au contacts. All $I-V$ curves were taken in air with a drain-source bias of $0.5 \mathrm{~V}$.

that produced by the spill-out surface charge and thus increases the $\mathrm{Au}$ work function. Furthermore, it is likely that $\mathrm{O}_{2}$ interacts directly with the SWNT-Au junction. The interaction of SWNTs with Au leads to the formation of an adsorption dipole and the generation of a local electric field. Such fields have been shown to enhance the adsorption of $\mathrm{O}_{2} \cdot{ }^{32,33} \mathrm{O}_{2}$ adsorbed at the SWNT-Au junction can now draw away electrons from the SWNT at the surface. The resulting energy-level alignment is shown in Figure 2B. By taking into account the above findings, we conclude that electrons are transferred from Au to the SWNTs in vacuum or oxygen-free environments, and adsorbate-mediated charge transfer in the opposite direction takes place in air. We note that previous discussions of charge transfer in the SWNT high-work-function metals ${ }^{4}$ such as Au assumed that electron transfer in the absence of oxygen proceeds from the nanotube to the metal. This conclusion was based on tabulated work functions of the individual components (metal, NT) without taking into account the interactions that take place upon adsorption. Our results show that such comparisons are inappropriate.

To test the role of local variations of the substrate work function on the energy-level alignments at the metal-NT interface further, we carried out SKPM and EFM measurements of SWNTs deposited on Au surfaces that had previously been passivated by being exposed to $\mathrm{H}_{2} \mathrm{~S}$ molecules and by covering the Au surface after SWNT deposition with an alkanethiol $\left(\mathrm{C}_{16} \mathrm{H}_{29} \mathrm{SH}\right) \mathrm{SAM}$. In contrast to unpassivated $\mathrm{Au}$ surfaces, the surface formed by exposure to $\mathrm{H}_{2} \mathrm{~S}$ (see Table 1) shows negligible CPDs between the adsorbed SWNTs and the sulfur-passivated Au surface; this is true in both vacuum and air. Furthermore, EFM measurements do not detect any significant charge transfer. This behavior can be understood by considering that the $S$ and $\mathrm{Au}$ atoms have essentially the same electronegativity, 2.5 vs 2.4, but the $\mathrm{S}$ atoms tie down the Au surface spill-out charge, generating a surface dipole that opposes the clean Au surface dipole and therefore reduces the surface work function. SWNTs interact weakly with this passivated surface, and EFM shows no charge transfer, so the Au Fermi level aligns near the middle of the SWNT band gap (see Figure 2C). The fact that the presence or absence of oxygen on the SWNT does not affect the CDP or the charge transfer is further proof that oxygen does not dope the nanotube but instead interacts with the gold substrate and the goldnanotube interface.

In the case of the samples involving SWNTs and alkanethiol coadsorption, we observe only small CPDs between SWNTs/Au and the alkanethiol SAMs/Au sites of $-0.03 \pm$ $0.02 \mathrm{~V}$ in UHV and $-0.05 \pm 0.02 \mathrm{~V}$ in air, respectively. The absence of an $\mathrm{O}_{2}$ effect is not surprising because the SAM inhibits oxygen access to the Au surface. Although the CDPs measured on the SAM-passivated surface are similar to those on the S/Au surface, the structures of the two surfaces are very different, suggesting that different interactions are measured. Considering the fact that alkanethiol SAMs are known to decrease the Au work function by as much as $1 \mathrm{eV}^{34}$ (we measured a $-0.5 \mathrm{~V}$ CPD between bare $\mathrm{Au}$ and the alkanethiol SAM-covered Au surface), the small differences in CDPs and the very weak interface dipole difference indicate that the vacuum-level difference between SWNTs and Au must be significantly increased compared to the difference of $0.08 \mathrm{~V}$ for the untreated surface in air. The dipole field of the SAM layer is similar to that of the SWNTs. Thus, the valence band of the SWNTs is shifted toward the Au Fermi level in the case of the thiol-passivated Au surface (see Figure 2D).

The above findings suggest that we can control the energylevel alignments at the source-SWNT and drain-SWNT junctions through coadsorption at the metal electrodes. This affects the carrier injection barriers of SWNT-FETs and therefore their electrical transport properties. First principles theoretical studies of coadsorption on small molecule/metal junctions have revealed similar effects. ${ }^{35}$ Moreover, these calculations show that, in addition to local work-function changes, more complex short-range interactions between the molecular wire and the coadsorbates can influence the transport properties of molecular wires.

In view of these strong interface effects, let us now consider the electrical transport characteristics of a SWNT- 
FET before and after the coadsorption of an alkanethiol SAM on the Au electrodes. An important characteristic of a FET is the subthreshold slope $S \equiv\left(\mathrm{d} \log I_{\mathrm{d}} / \mathrm{d} V_{\mathrm{g}}\right)^{-1}$, where $I_{\mathrm{d}}$ is the drain current and $V_{\mathrm{g}}$ is the gate voltage. $S$ is a measure of the switching speed of the device. ${ }^{36}$ Figure $3 \mathrm{~A}$ shows that $S$ for this p-type of SWNT-FET is initially large (i.e., shows poor switching), about $2 \mathrm{~V} /$ decade. However, upon SAM coadsorption, the subthreshold slope improves significantly to about $250 \mathrm{mV} / \mathrm{dec}$ ade. Moreover, the ratio of the $\mathrm{ON}$ state drain current to the OFF-state drain current of the FET improves by at least 1 order of magnitude. These effects can be understood in terms of the movement of the electrode Fermi level closer to the SWNT valence band, as discussed above, which reduces the Schottky barrier at the source/ SWNT junction and enhances hole injection..$^{8,11-14}$ As expected, however, the effect of sulfur passivation is quite different. Figure 3B shows the characteristics of a device obtained after sulfur atom prepassivation of the Au electrode surface. This treatment leads to an ambipolar device (i.e., one in which both electron (hole) transport is possible with positive (negative) gate bias, even in air with a yield $>0.1$ ). This result is in accord with the findings above that the Au Fermi level is aligned with the mid-gap of the NT and that there is no charge transfer between the SWNT and the sulfurpassivated surface. Thus, coadsorption and passivation provides a novel and powerful way of controlling the junction properties and, therefore, the performance of SWNT-FETs. This is in contrast to conventional $\mathrm{Si}$ devices where such control requires doping.

In conclusion, we have used scanning Kelvin probe and electrostatic force microscopies to study the charge-transfer processes at the $\mathrm{Au}$-carbon nanotube junctions and have correlated these findings with the transport characteristics of nanotube field-effect transistors. We found that properties of the metal electrode/nanotube interface control the characteristics of the nanotube FETs, a fact that is consistent with the proposed Schottky barrier model for the SWNT-FETs. The SWNT-FET properties can be changed by coadsorption or passivation of the metal/SWNT interface with appropriate atoms or molecules. The resulting modifications of the Schottky barriers provide a powerful way of tuning the transistor characteristics. Similar effects are expected in small-molecule transistors.

Acknowledgment. We thank J. Bucchignano for help with the e-beam lithography, B. Ek, V. Derycke, and L. Chen for experimental assistance, and J. Appenzeller and O. Cherniavskaya for helpful discussions. This work was supported by the NSF under Columbia Nanocenter grant CHE-01-17752. The EFM facilities are partially supported by MRSEC grant DMR-0213574.

\section{References}

(1) Carbon Nanotubes: Synthesis, Structure, Properties and Applications; Dresselhaus, M. S., Dresselhaus, G., Avouris, Ph., Eds.; SpringerVerlag: Berlin, 2001.

(2) McEuen, P.; Fuhrer, M. S.; Park, H. IEEE Trans. Nanotech. 2002 1,78 .

(3) Appenzeller, J.; Joselevich, E.; Hoenlein, W. Carbon Nanotubes for Data Processing in Nanoelectronics and Information Technology; Wiley-VCH Verlag: Berlin, 2003.

(4) Tans, S. J.; Verschueren, A. R. M.; Dekker, C. Nature 1998, 393, 49.

(5) Martel, R.; Schmidt T.; Shea H. R.; Hertel T.; Avouris Ph. Appl. Phys. Lett. 1998, 73, 2447.

(6) Zhou, C.; Kong, J.; Dai, H. Appl. Phys. Lett. 1999, 76, 1597.

(7) Derycke, V.; Martel, R.; Appenzeller, J.; Avouris, Ph. Appl. Phys. Lett. 2002, 80, 2773

(8) Derycke, V.; Martel, R.; Appenzeller, J.; Avouris, Ph. Nano Lett. 2001, 1,453 .

(9) Collins, P. G.; Bradley, K.; Ishigami, M.; Zettl, A. Science 2000, 287, 1801.

(10) Jhi, S.-H.; Louie, S. G.; Cohen, M. Phys. Rev. Lett. 2000, 85, 1710.

(11) Martel, R.; Derycke, V.; Lavoie, C.; Appenzeller, J.; Chan, K. K.; Tersoff, J.; Avouris, Ph. Phys. Rev. Lett. 2001, 87, 256805-256809.

(12) Freitag, M.; Radosavljevic, M.; Zhou, Y. X.; Johnson, A. T.; Smith, W. F.; Appl. Phys. Lett. 2001, 79, 3326.

(13) Appenzeller, J.; Knoch, J.; Derycke, V.; Martel, R.; Wind, S.; Avouris, Ph. Phys. Rev. Lett. 2002, 89, 126801.

(14) Heinze, S.; Tersoff, J.; Martel, R.; Derycke, V.; Appenzeller, J.; Avouris, Ph. Phys. Rev. Lett. 2002, 89, 106801.

(15) Nakanishi, T.; Bachtold, A.; Dekker: C Phys. Rev. B 2002, 66, 073303.

(16) Nonnenmacher, M.; O’Boyle, M. P.; Wickramasinghe, H. K. Appl. Phys. Lett. 1991, 58, 2921.

(17) Martin, Y.; Abraham, D. W.; Wickramasinghe, H. K. Appl. Phys. Lett. 1988, 52, 1103.

(18) Thess, A.; Lee, R.; Nikolaev, P.; Dai, H.; Petit, P.; Robert, J.; Xu, C.; Lee, Y. H.; Kim, S. G.; Rinzler, A. G.; Colbert, D. T.; Scuseria, G. E.; Tomanek, D.; Fischer, J. E.; Smalley, R. E. Science 1996, $273,483$.

(19) Bachilo, S. M.; Strano, M. S.; Kitrell, C.; Hauge, R. H.; Smalley, R. E.; Weisman, R. B. Science 2002, 298, 2361.

(20) Hagen, A.; Hertel, T. Nano Lett. 2003, 3, 383.

(21) Cherniavskaya, O.; Chen, L. W.; Weng, V.; Yuditsky, L.; Brus, L. E. J. Phys. Chem. B 2003, 107, 1525 .

(22) Touzov, I.; Gorman, C. B. Langmuir 1997, 13, 4850

(23) Suzuki, S.; Bower, C.; Watanabe, Y.; Zhou, O. Appl. Phys. Lett. 2000, 76, 4007-4010.

(24) Ulbricht, H.; Moos, G.; Hertel, T. Phys. Rev. B 2002, 66, 75404:1.

(25) Sorescu, D. C.; Jordan, K. D.; Avouris, Ph. J. Phys. Chem. B 2001, $105,11227$.

(26) Potter, H. C.; Blakeley, J. M. J. Vac. Sci. Technol. 1975, 12, 635.

(27) Sugiyama, K.; Ishii, H.; Ito, E.; Seki, K. Adv. Mater. 1999, 11, 605.

(28) Hertel, T.; Walkup, R. E.; Avouris, Ph. Phys. Rev. B 1998, 58, 13870.

(29) Heeres, A.; Veenstra, S. C.; Hadziioannou, G.; Sawatzky, G. A.; Jonkman, H. T. Appl. Phys. A 2002, 75, 661.

(30) Odom, T. W.; Kim, P.; Huang, J.-L.; Lieber, C. M. Phys. Rev. Lett. 1999, 82, 1225.

(31) Koch, N.; Kahn, A.; Ghijsen, J.; Pireaux, J.-J.; Schwartz, J.; Johnson, R. L.; Elschner, A. Appl. Phys. Lett. 2003, 82, 70.

(32) Mihaychuk, J. G.; Shamir, N.; van Driel, H. M. Phys. Rev. Lett. 1999, $82,359$.

(33) Similarly, electric fields produced by oxide charges or the gate field can enhance the adsorption of $\mathrm{O}_{2}$ in NT-FET devices.

(34) Schoenenberger, C.; Jorritsma, J. C.; Sondag-Huethorst, J. A. M.; Fokkink, L. G. J. J. Phys. Chem. 1995, 99, 3259.

(35) Lang, N. D.; Avouris, Ph. Nano Lett. 2002, 2, 1047.

(36) Sze, S. M. Physics of Semiconductor Devices; Wiley: New York, 1981.

NL034193A 